\title{
Proximal femur lag screw placement based on bone mineral density determined by quantitative computed tomography
}

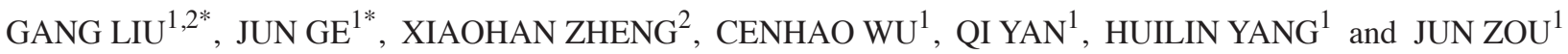 \\ ${ }^{1}$ Department of Orthopaedic Surgery, The First Affiliated Hospital of Soochow University, Suzhou, Jiangsu 215006; \\ ${ }^{2}$ Department of Emergency Medicine, The Affiliated Hospital of Guizhou Medical University, Guiyang,
} Guizhou 550001, P.R. China

Received June 7, 2019; Accepted October 22, 2019

DOI: $10.3892 /$ etm. 2020.8480

\begin{abstract}
Following internal fixations for intertrochanteric fractures in elderly patients, lag screws or screw blades frequently cut the femoral head, leading to surgical failure. The bone mineral density (BMD) at various parts of the proximal femur is significantly correlated with the holding force of the lag screw, which in turn is closely associated with the stability of the fixation. However, the appropriate placement of the lag screw has been controversial. As a novel detection method for $\mathrm{BMD}$, quantitative computed tomography (QCT) may provide relatively accurate measurements of three-dimensional structures and may provide an easy way to determine the appropriate lag screw placement. A total of 50 elderly patients with intertrochanteric fractures were selected for the present study. The BMD of the proximal femur on the healthy side, including the femoral intertrochanter, neck and head, was measured using QCT. For testing, the femoral head was divided into medial, central and lateral sections. The BMD of the femoral head was determined to be the highest, while the BMD of the femoral neck was the lowest. In the femoral head, the central section had the highest BMD, while the lateral section had the lowest BMD. The present study used QCT to detect differences in the BMD at various regions of the proximal femur and provided a novel theoretical reference for the placement of lag screws. To obtain maximum holding power, the lag screw must be placed in the central section of the femoral head.
\end{abstract}

Correspondence to: Dr Jun Zou or Dr Huilin Yang, Department of Orthopaedic Surgery, The First Affiliated Hospital of Soochow University, 188 Shizi Street, Suzhou, Jiangsu 215006, P.R. China E-mail: jzou@suda.edu.cn

E-mail: hlyang@suda.edu.cn

*Contributed equally

Abbreviations: PFNA, proximal femoral nail anti-rotation; BMD, bone mineral density; QCT, quantitative computed tomography

Key words: intertrochanteric fractures, internal fixation, bone mineral density, quantitative computed tomography

\section{Introduction}

As aging increases in society, the incidence of proximal femoral fractures in the elderly, particularly intertrochanteric fractures, has seen an increase over the years. Complications arising from these fractures, including cardiovascular and cerebrovascular diseases and lung infections, threaten the health of these patients (1). To reduce such complications, the AO Foundation recommends intramedullary fixation, including proximal femoral nail anti-rotation (PFNA), InterTan, and other internal fixation methods, for the treatment of intertrochanteric fractures. These methods effectively increase the force arm of the fixation device. The lag screw or screw blade is inserted into the femoral head through the femoral neck and intertrochanter; this effectively increases the firmness of the fixation, particularly for osteoporotic fragility fractures (2). However, in clinical practice, the screw may loosen, and in certain cases, the femoral head is cut off, resulting in surgical failure (3). Recent studies have indicated that the bone mineral density (BMD), bone microstructural parameters and poor placement of screws in the femoral head are significantly associated with surgical failure (4,5). Among these, the bone microstructure of the femoral head is the most important factor; in particular, tension and femoral head pressure of trabecular bones are important and have a significant guiding function for selection of surgical techniques (6).

Although the former studies have highlighted the important association between the femoral head density and the success of internal fixation, the roles of the BMD of the femoral head's medial, central and lateral portions have remained to be fully explored, which make the placement of screws a challenging task for surgeons.

To improve the success rate of internal fixation, the present study aimed to examine the BMD of the proximal part of the femur and provide a theoretical basis for the optimal placement of the lag screw.

\section{Materials and methods}

Patient information. From January 2017 to October 2018, 50 patients with senile femoral intertrochanteric fractures were selected from the Department of Trauma and Orthopedics, The Affiliated Hospital of Guizhou Medical University, (Guizhou, 
China). Cases with developmental dysplasia of the hip, femoral head necrosis, pathological fractures and primary hip fusion were excluded.

The BMD of the proximal part of the injured femur was measured using quantitative computed tomography (QCT). Among the patients, 10 were males and 40 were females, all aged between 60 and 97 years, with a mean age of 78.02 years. The AO classification was 31A1.1-3 type in 13 cases, 31A2.1-3 type in 21 cases and 31A3.1-3 type in 16 cases. The present study was approved by the ethics committee of The Affiliated Hospital of Guizhou Medical University, (Guizhou, China). Informed consent was obtained from all patients and their families.

Measurement area selection. To determine the detection area, six lines were drawn as follows (Fig. 1): 'line a', a straight line through the longitudinal axis of the femoral neck and the center of the femoral head; 'line b', a straight line through the midpoint of the femoral head, perpendicular to 'line a'; 'line c', the line drawn through the femoral intertrochanter, perpendicular to 'line a' (the detection area is selected from the center of the line, which represents the intertrochanteric BMD); 'line d', the line formed perpendicular to 'line a' through the middle of the femoral neck (the detection area is selected from the center of the line, which represents the BMD of the femoral neck); 'line e', a straight line formed perpendicular to 'line b' through three equal points on the outer side of the femoral head (the detection area is selected from the center of the line, representing the BMD outside the femoral head); and 'line f', the line formed perpendicular to 'line b' through three equal points on the inner side of the femoral head (the detection area is selected from the center of the femoral head, representing the BMD of the internal femoral head). To represent the BMD of the central section of the femoral head, the detection area was selected from 'line b'.

Measurement methods. The SIEMENS 64-row SOMATOM Definition AS and spiral CT scanner (Siemens AG) were used to scan the bilateral hip bone structures of the patients, including the acetabular and femoral proximal bone structures. The original images of the contralateral hip, including the femoral intertrochanter, neck and head, were obtained. These images were 400 Hounsfield units (HU) wide, with a window level of $40 \mathrm{HU}$, a layer thickness of $1 \mathrm{~mm}$ and an interval of $1 \mathrm{~mm}$. The QCT Pro software's 'QA Exam' function was used to calibrate the software accuracy and the scan field uniformity of the CT equipment. The corresponding scan field uniformity calibration coefficient and QA phantom calibration data were obtained to ensure the accuracy of the analysis software. The raw images of the contralateral side were taken from a picture archiving and communication system (SIEMENS syngo.plaza; Siemens AG) and the CT values of the intertrochanteric bone and the femoral head and neck were measured. The Mindways quantitative QCT BMD measurement software (QCT Pro 4.2.3; Mindways Software, Inc.) was used to analyze the test data. When the femoral head was detected, it was longitudinally divided into three sections: The outer, central and inner sections. Each section was tested thrice, and each time, a circular test area of a fixed size $\left(2.43 \mathrm{~cm}^{2}\right)$ was selected. The mean value was recorded as the final test result and expressed in $\mathrm{mg} / \mathrm{cm}^{3}$ (Fig. 1).
Statistical analyses. Statistical analyses were performed using SPSS software (version 16.0, SPSS, Inc.). First, the Kolmogorov-Smirnov test was performed to determine whether the data were normally distributed. If they were identical, one-way analysis of variance (ANOVA) with Bonferroni's post-hoc test was performed to compare the differences. If they were not identical, the Kruskal-Wallis test with Steel-Dwass post-hoc test was used to compare the differences. The Brown-Forsythe or the Welch values were analyzed instead of the P-values in case of variance; significance values of $<0.05$ were considered to indicate a significant difference.

\section{Results}

Heterogeneity test. The Kolmogorov-Smirnov test indicated that the homodyne data of the femoral neck, femoral intertrochanter and each of the three parts of the femoral head were identical. However, data of the femoral head was not normally distributed. Therefore, one-way ANOVA was used to compare the differences between the femoral neck and head, and among the three sections of the femoral head. The Kruskal-Wallis test was used for comparison between femoral head and neck, femoral head and intertrochanter.

BMD analysis. The BMD of the femoral head was the highest, followed by that of the femoral intertrochanter, and the BMD of the femur neck was the lowest (Fig. 2). These differences were statistically significant $(\mathrm{P}<0.01)$. Among the femoral head sections, $\mathrm{BMD}$ analysis revealed that the lateral and the inner sections were similar $(\mathrm{P}>0.05)$. The results suggested that the $\mathrm{BMD}$ of the central section was the largest and the medial and lateral bone densities were significantly smaller $(\mathrm{P}<0.05$; Fig. 3). In summary, the tensile trabecular bone density was significantly less than the pressure trabecular bone density $(\mathrm{P}<0.05)$.

\section{Discussion}

The high surgical failure of proximal femoral fractures has drawn public attention. Patients with fixation failures are more likely to suffer a reduction in the quality of life upon discharge with a consequent increase in social dependency (7). There are various reasons for the high incidence and the device design is mostly accountable. Born et al (8) reported that the small-contact interface between the PFNA spiral blade and the femoral skull, at $75 \mathrm{~cm}^{2}$, is not conducive for riveting between the internal fixation device and the femoral skull. A biomechanical study by Bonnaire et al (9) suggested that a novel design of the central loading device may increase the load-bearing capacity and thus help to reduce the cutting-out phenomenon. More importantly, surgeon-dependent factors, including suboptimal positioning of the device, has a significant role in fixation failure (10). Except for the above reasons, an increasing amount of studies have revealed that BMD is highly positively correlated with the stability of the internal fixation. Konstantinidis et al (11) indicated that when the femoral head volumetric BMD (vBMD) was $<250 \mathrm{mg} / \mathrm{cm}^{3}$, five out of nine patients developed a screw cut, but when the vBMD was $>250 \mathrm{mg} / \mathrm{cm}^{3}$, only 1 of 21 patient developed a screw cut. When the BMD decreases, the structural model 


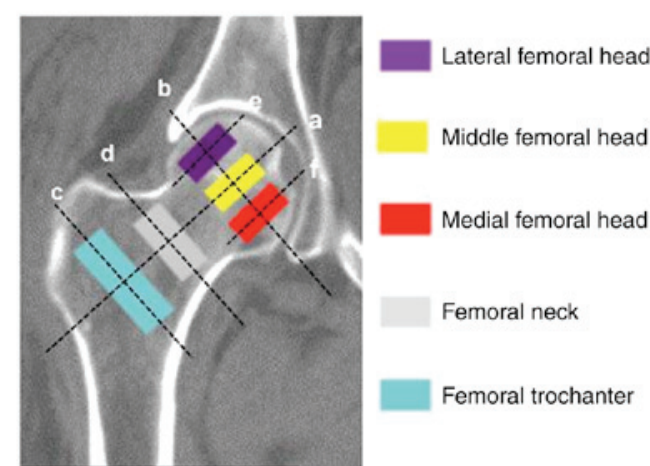

Figure 1. Six lines were drawn to determine the detection area.

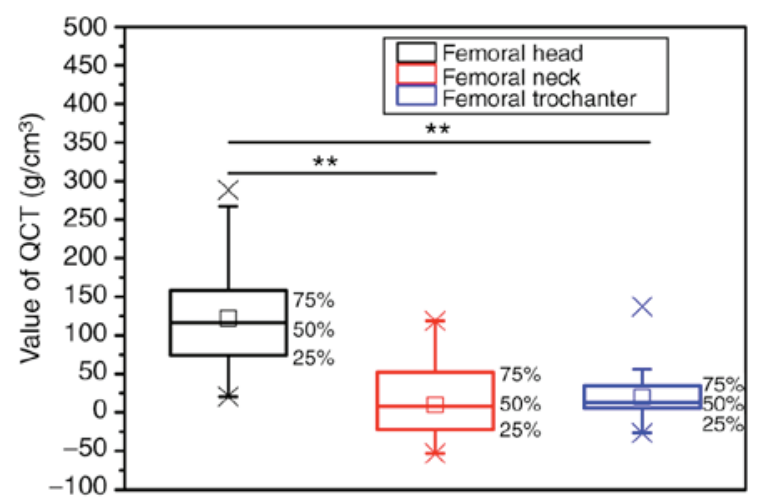

Figure 2. Box plot of the femoral BMD. The BMD of the femoral head was the highest, followed by the BMD of the femoral intertrochanter; the BMD of the femoral neck was the lowest among the three. These differences were statistically significant. ' $\square$ ' represents the mean of the sample. The line in the middle of the box and the ' $50 \%$ ' beside it represents the median. The upper and lower margins in the box and the ' 25 ' and ' $75 \%$ ' beside them represent quartiles. ' $x$ ' represents the extreme values. The upper and lower margin of the line through represents 1.5 times the interquartile range beyond the upper and lower quartile. ${ }^{* *} \mathrm{P}<0.01$. BMD, bone mineral density; QCT, quantitative computed tomography.

index (SMI) increases, the trabecular bone changes in shape from a plate to a rod and the fixation strength of the intraosseous screw is weakened. Kang et al (12) performed a multiple linear regression analysis, indicating that the SMI is negatively correlated with the stability of the embedded internal fixation. Further research has revealed that lag screw placement in the femoral head is closely associated with the stability of the internal fixation device.

To prevent the screw from being cut off, certain researchers have advocated placing the lag screw under the femoral head. Through biomechanical studies and finite element analyses, Kuzyk et al, Goffin et al and Bessho et al (13-15) revealed that when the lag screw was located below the center of the femoral neck, greater axial compression, torsional shear resistance and minimal load deformation were observed, indicating that this position was the most conducive for internal fixation. However, even when surgeons strictly follow these techniques, internal fixation failure cannot be avoided. Therefore, in the present study, the BMD was used as a decisive factor, and the BMD of the femoral intertrochanter, neck and head in elderly patients with intertrochanteric fractures were measured. The femoral

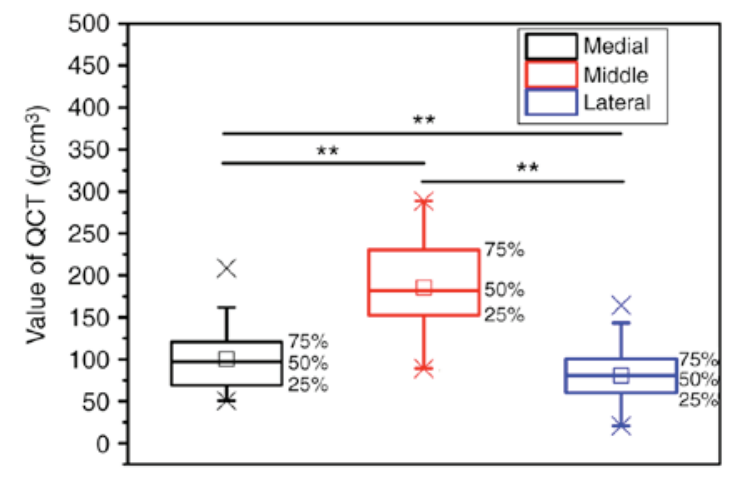

Figure 3. Bone mineral density of the three sections of the femoral head revealed that the lateral part was similar to the inner part $(\mathrm{P}>0.05)$. The medial and lateral bone densities were significantly lower than that of the central section $(\mathrm{P}<0.05)$. ' $\square$ ' represents the mean of the sample. The line in the middle of the box and the ' $50 \%$ ' beside it represents the median. The upper and lower margins in the box and the ' 25 ' and ' $75 \%$ ' beside them represent quartiles. ' $x$ ' represents the extreme values. The upper and lower margin of the line through represents the 1.5 times the interquartile range beyond the upper and lower quartile. ${ }^{* *} \mathrm{P}<0.01$. QCT, quantitative computed tomography.

head was further divided into three equal sections: The medial, central and lateral sections. Their BMDs were measured to provide a theoretical basis of the optimal position of the lag screws or spiral inserts of internal fixation devices, including the PFNA and InterTan. Numerous methods are available for detecting BMD; dual-energy X-ray absorptiometry is the most traditionally applied method, but its disadvantage is that it only provides the BMD of two-dimensional structures and cannot accurately and comprehensively display the complete BMD (16). A QCT, on the other hand, is able to detect the BMD of a three-dimensional structure more accurately (17). Although previous studies have used QCT to detect the BMD in patients with hip fractures, these studies have focused on the association between BMD and the incidence of proximal femoral fractures, without further investigation into their treatment (18-21).

The trabecular bone in the femoral head is divided into the pressure and tension trabecular bones. Pressure trabeculae grow mainly in the central and medial regions of the femoral head, from the lower side of the femoral neck to its medial side; these bear the pressure load. The tension trabeculae begin from the upper side of the femoral neck and grow to its inside and below the femoral head. Regarding the tension load, the pressure and tension trabecular bones cross each other in the central region of the femoral head, enhancing the weight-bearing function of the tension trabecular bone (22). Dendorfer et al (23) indicated that the compressive elastic modulus, ultimate strength and yield strength of the pressure trabecular bone were larger than those of the tensile trabecular bone.

First, the present study indicated that, among the BMDs of the three proximal femur portions, that of the femoral neck is the lowest. This may be due to the presence of the Ward's triangle in the femoral neck, consisting of the tension trabecular bone, the pressure trabecular bone and the gap formed by the partial tension trabecular bone, all of which are mainly filled by fatty bone marrow. The mean BMD between the femoral trochanters was slightly higher than that of the femoral neck, 
as the intertrochanteric region is mainly composed of partial pressure trabecular bone and the femur. As no obvious cavity was present, it may be hypothesized that the grip force of the screw on the femoral intertrochanter was greater than that on the femoral neck. This suggests the importance of bone integrity of the femoral intertrochanter in fracture fixation. If no appropriate surgical method is selected, the internal fixation may fail, as the BMD of the intertrochanter may be too low, or the outer wall of the interior rotor may break, resulting in reduction of the screw's holding force. The BMD between the femoral intertrochanter and neck was significantly less than that of the femoral skull, with the BMD of the latter being equivalent to the mean densities of the medial, central and lateral sections. This is mainly due to the femoral head being composed of the pressure and tension trabecular bones with no cavity, and the long-term pressure and tensile loads cause the femoral head BMD to be significantly higher than that of the other two. Since BMD appeared to be positively correlated with the stability of the internal fixation, this observation indicates that the femoral head has the highest holding force on the lag screw. Therefore, when the fracture is fixed internally, the screw that fixes the fracture must reach the femoral head to obtain the maximum grip force and reduce the risk of failure. This is consistent with the results of a mechanical study by Baumgaertner et al (24) on BMD, i.e., the screw-fixed apex moment must be $<25 \mathrm{~mm}$ to achieve the optimal fixation effect.

A comparative analysis of the BMD of the medial, central and lateral trabecular bones of the femoral head revealed that the mean BMD of the lateral part was lower than that of the medial part. This is due to the presence of a portion of the tension trabecular bone in the medial side of the femoral head. This bone has a lesser load on the hip than the pressure trabecular bone, as the lower limb mainly has a bearing role, resulting in a lower skeletal BMD of the tension trabecular bone than that of the pressure trabecular bone (25). However, this difference was not statistically significant, indicating that the holding force generated by the two parts of the screw was not significantly different. This demonstrates that the lag screw mainly has a supporting role. Therefore, a deeper placement of the lag screw (deeper than the biomechanical requirement) inside the femoral head may be recommended. The BMD of the two other regions of the femoral head was significantly lower than that of the trabecular bone in the central section. This is due to the femoral head pressure and tension trabecular bones intersecting in this area, where the pressure trabecular bone is mainly located, resulting in the highest skeletal BMD in the central part (26). This indicates that during internal fixation of fractures, the screw is best placed in the central section of the femoral head, where it is able to obtain the maximum holding force.

Although the present study performed BMD measurements of the proximal femoral structures and a theoretical analysis of the holding power of the screw, it still has certain shortcomings. The overlapping tension and pressure trabecular bones in the central region of the femoral head cannot be well-distinguished from an anatomical point of view. Furthermore, due to the fracture, it was not possible to measure the BMD of the proximal femur on the injured side; only the BMD on the healthy side was determined. Finally, post-operative follow-up was not performed in the present study. These points therefore require further investigation.

In conclusion, the location of internal fixation in proximal femoral fractures cannot be determined using a single method, and it is required to consider various factors. Therefore, the present study used QCT to detect differences in the BMD in various regions of the proximal femur and provided a novel theoretical reference for the placement of lag screws.

\section{Acknowledgements}

Not applicable.

\section{Funding}

This study was supported by the National Natural Science Foundation of China (grant nos. 81472132, 81572183, 81672220 and 91849114) and the Priority Academic Program Development of Jiangsu Higher Education Institutions.

\section{Availability of data and materials}

The datasets used and/or analyzed during the present study are available from the corresponding author on reasonable request.

\section{Authors' contributions}

GL, JG, XZ, CW and QY collected the QCT data and searched the literature. GL, JG, HY and JZ analyzed and interpreted the data. GL and JG assembled the figures and wrote the manuscript. HY and JZ designed the study and obtained the funding. All authors read and approved the final manuscript.

\section{Ethics approval and consent to participate}

All of the experimental procedures were approved by the Ethics Committee of the First Affiliated Hospital of Soochow University (Suzhou, China) and were in strict accordance with Declaration of Helsinki (1964). Informed consent to participate in the study was obtained from the patients and their families.

\section{Patient consent for publication}

Not applicable.

\section{Competing interests}

The authors declare that they have no competing interests.

\section{References}

1. Mundi S, Pindiprolu B, Simunovic N and Bhandari M: Similar mortality rates in hip fracture patients over the past 31 years. Acta Orthop 85: 54-59, 2014.

2. Kristek D, Lovric I, Kristek J, Biljan M, Kristek G and Sakic K: The proximal femoral nail antirotation (PFNA) in the treatment of proximal femoral fractures. Coll Antropol 34: 937-940, 2010.

3. Steiner JA, Ferguson SJ and Van Lenthe GH: Computational analysis of primary implant stability in trabecular bone. J Biomech 48: 807-815, 2015. 
4. Grechenig S, Gänsslen A, Gueorguiev B, Berner A, Müller M, Nerlich M and Schmitz P: PMMA-augmented SI screw: A biomechanical analysis of stiffness and pull-out force in a matched paired human cadaveric model. Injury 46 (Suppl 4): S125-S128, 2015.

5. Schiuma D, Plecko M, Kloub M, Rothstock S, Windolf M and Gueorguiev B: Influence of peri-implant bone quality on implant stability. Med Eng Phys 35: 82-87, 2013.

6. Van Rietbergen B, Van Huiskes R, Eckstein F and Rüegsegger P: Trabecular bone tissue strains in the healthy and osteoporotic human femur. J Bone Miner Res 18: 1781-1788, 2010.

7. Broderick JM, Bruce-Brand R, Stanley E and Mulhall KJ Osteoporotic hip fractures: The burden of fixation failure ScientificWorldJournal 2013: 515197, 2013.

8. Born CT, Karich B, Bauer C, von Oldenburg G and Augat P: Hip screw migration testing: First results for hip screws and helical blades utilizing a new oscillating test method. J Orthop Res 29: 760-766, 2011.

9. Bonnaire F, Weber A, Bösl O, Eckhardt C, Schwieger K and Linke B: 'Cutting out' in pertrochanteric fractures-problem of osteoporosis?. Unfallchirurg 110: 425-432, 2007 (In German).

10. Audigé L, Hanson B and Swiontkowski MF: Implant-related complications in the treatment of unstable intertrochanteric fractures: Meta-analysis of dynamic screw-plate versus dynamic screw-intramedullary nail devices. Int Orthop 27: 197-203, 2003.

11. Konstantinidis L, Papaioannou C, Blanke P, Hirschmüller A, Südkamp N and Helwig P: Failure after osteosynthesis of trochanteric fractures. Where is the limit of osteoporosis? Osteoporos Int 24: 2701-2706, 2013.

12. Kang SR, Bok SC, Choi SC, Lee SS, Heo MS, Huh KH, Kim TI and Yi WJ: The relationship between dental implant stability and trabecular bone structure using cone-beam computed tomography. J Periodontal Implant Sci 46: 116-127, 2016.

13. Kuzyk PR, Zdero R, Shah S, Olsen M, Waddell JP and Schemitsch EH: Femoral head lag screw position for cephalomedullary nails: A biomechanical analysis. J Orthop Trauma 26: 414-421, 2012.

14. Goffin JM, Pankaj P and Simpson AH: The importance of lag screw position for the stabilization of trochanteric fractures with a sliding hip screw: A subject-specific finite element study. J Orthop Res 31: 596-600, 2013.

15. Bessho M, Ohnishi I, Matsumoto T, Ohashi S, Matsuyama J, Tobita K, Kaneko M and Nakamura K: Prediction of proximal femur strength using a CT-based nonlinear finite element method: Differences in predicted fracture load and site with changing load and boundary conditions. Bone 45: 226-231, 2009.
16. Watts NB: Fundamentals and pitfalls of bone densitometry using dual-energy X-ray absorptiometry (DXA). Osteoporos Int 15 : 847-854, 2004

17. Shim VB, Pitto RP and Anderson IA: Quantitative CT with finite element analysis: Towards a predictive tool for bone remodelling around an uncemented tapered stem. Int Orthop 36: 1363-1369, 2012.

18. Black DM, Bouxsein ML, Marshall LM, Cummings SR, Lang TF, Cauley JA, Ensrud KE, Nielson CM and Orwoll ES; Osteoporotic Fractures in Men (MrOS) Research Group: Proximal femoral structure and the prediction of hip fracture in men: A large prospective study using QCT. J Bone Miner Res 23: 1326-1333, 2010

19. Cheng X, Li J, Lu Y, Keyak J and Lang T: Proximal femoral density and geometry measurements by quantitative computed tomography: Association with hip fracture. Bone 40: 169-174, 2007.

20. Cheng XG, Lowet G, Boonen S, Nicholson PH, Brys P, Nijs J and Dequeker J: Assessment of the strength of proximal femur in vitro: Relationship to femoral bone mineral density and femoral geometry. Bone 20: 213-218, 1997.

21. Cody DD, Divine GW, Nahigian K and Kleerekoper M: Bone density distribution and gender dominate femoral neck fracture risk predictors. Skeletal Radiol 29: 151-161, 2000.

22. Cui WQ, Won YY, Baek MH, Lee DH, Chung YS, Hur JH and Ma YZ: Age-and region-dependent changes in three-dimensional microstructural properties of proximal femoral trabeculae. Osteoporos Int 19: 1579-1587, 2008.

23. Dendorfer S, Maier HJ, Taylor D and Hammer J: Anisotropy of the fatigue behaviour of cancellous bone. J Biomech 41: 636-641, 2008.

24. Baumgaertner MR, Curtin SL, Lindskog DM and Keggi JM: The value of the tip-apex distance in predicting failure of fixation of peritrochanteric fractures of the hip. J Bone Joint Surg Am 77: 1058-1064, 1995

25. Jang IG and Kim IY: Computational study of wolff's law with trabecular architecture in the human proximal femur using topology optimization. J Biomech 41: 2353-2361, 2008.

26. Li B and Aspden RM: Material properties of bone from the femoral neck and calcar femorale of patients with osteoporosis or osteoarthritis. Osteoporos Int 7: 450-456, 1997. 